\title{
The Little Miss Muffet Effect: Quantifying the Effect of Predation Risk on Foraging Patch Choice by Houseflies (Musca domestica)
}

\author{
Colleen Riley ${ }^{1}$ and Lawrence M. Dill ${ }^{1,2}$
}

Accepted July 13, 2005; revised July 20, 2005

The ability of the ideal free distribution (IFD) to predict patch choice of female houseflies (Musca domestica) was determined by examining their distribution between two patches containing unequal amounts of food. The effect of predation risk was then quantified in energetic terms by examining fly distribution between patches of equal food, with one containing spiders. Results were used to predict how much extra food must be added to the risky patch to offset the risk of predation. Flies were found to conform fairly closely to the IFD. Predation risk had a major effect on their distribution, with fewer flies feeding in the presence of predators as risk increased. Addition of extra food to the risky patch was successful in offsetting the risk of predation. These results suggest that the effect of risk on housefly foraging behavior can be quantified in energy terms, providing a common currency for predicting the effects of resources and predation risk on habitat use.

KEY WORDS: ideal free distribution; predation risk; titration; houseflies; spiders.

\section{INTRODUCTION}

Optimal foraging theory (MacArthur and Pianka, 1966; Stephens and Krebs, 1986) predicts that animals weigh the costs and benefits associated with different patches when making foraging decisions, in order to

\footnotetext{
${ }^{1}$ Behavioral Ecology Research Group, Department of Biological Sciences, Simon Fraser University, 8888 University Drive, Burnaby, BC V5A 1S6 Canada.

${ }^{2}$ To whom correspondence should be addressed. E-mail: ldill@sfu.ca.
} 
maximize fitness. When decisions are made purely from a resource perspective, animals are predicted to choose patches that maximize their energy intake. However, predation risk can have a profound effect on the fitness value of a patch and thus on foraging decisions (reviewed by Lima and Dill, 1990). In order to quantify the interacting effects of food availability and predation risk on foraging decisions, these factors ideally should be assessed in terms of their effects on fitness. Because fitness is difficult to measure, several studies have demonstrated that these factors can be assessed in a surrogate common currency by determining the energetic equivalence of predation risk (Abrahams and Dill, 1989; Kotler and Blaustein, 1995; Abramsky et al., 2002).

Ideal free distribution (IFD) theory (Fretwell and Lucas, 1970) describes how a group of animals should distribute themselves among habitat patches such that all individuals receive equal fitness benefits. This model assumes that animals are "ideal" in their knowledge of the environment, and "free" to move between patches and access resources. When these assumptions are met, IFD theory predicts that a group of foraging animals will distribute themselves among patches such that the proportion of individuals in each patch matches the proportion of resources available there. In this way, each individual is able to obtain energy at the same rate. Conformity to the ideal free distribution has been documented in a number of animals including ants (Lamb and Ollason, 1993), daphnids (Larsson, 1997), guppies (Abrahams, 1989), sticklebacks (Gotceitas and Colgan, 1991), tits (Diaz et al., 1998), and even humans (Kraft and Baum, 2001; Madden et al., 2002). In addition, several species of insects have been shown to conform to the IFD when searching for mates, including dung flies (Parker, 1978) and butterflies (Courtney and Parker, 1985).

If a group of animals conforms to IFD, deviations from this distribution in the presence of predators allows for the quantification of predation risk in terms of energy. IFD predicts that animals should distribute themselves equally among two patches with equal amounts of food. However, if one patch has an associated risk of predation, fewer individuals are predicted to feed in this risky patch than in the safe alternative. Individuals in the risky patch will have access to a greater amount of energy than those feeding in the safe patch, but risk the fitness costs of predation. Assuming IFD, animals in both patches will have equal expected fitness. The energy equivalence of predation risk is then the difference in energy intake between individuals in the risky and safe patches.

In this study, the effect of predation risk on the foraging decisions of female houseflies (Musca domestica Linnaeus) was determined as described above. First, we determined the validity of the ideal free distribution as a model for patch choice. Next, the effect of risk of spider predation on 
the distribution of flies among patches was determined. Results were then used to predict how much extra food must be added to a patch with an associated risk of predation in order to offset the cost of predation risk. This experiment provides a simple system for testing both IFD and the effects of predation risk on foraging decisions.

\section{METHODS}

Only female houseflies were used since sex-related difference have been found in previous IFD studies (Abrahams, 1989; Abrahams and Dill, 1989). All flies were between 5 and 8 days old at the time of experiments, but all those used within a single trial were of the same age. Flies were used only once. Flies were reared on a diet of sugar cubes and evaporated milk and starved for $24 \mathrm{~h}$ before trials. Experiments were performed in a glass tank measuring $42.5 \mathrm{~cm} \times 22.3 \mathrm{~cm} \times 28.2 \mathrm{~cm}(\mathrm{~L} \times \mathrm{W} \times \mathrm{H})$. The tank was divided into two equal patches by a white ceramic tile that extended $15.5 \mathrm{~cm}$ up from the bottom of the tank, allowing flies to move freely between the two patches. The top of the tank was covered in wire mesh with stocking-covered holes above each patch that allowed flies to be released into the tank without escaping. One soaked cotton ball provided water in each patch in all trials. Sugar cubes were placed on the aquarium floor, in the centre of each patch.

In each trial, 30 flies were released into the apparatus and patch use was recorded for $1 \mathrm{~h}$ This length of time was chosen because under these experimental conditions, flies began to lose interest in feeding after this time. The tank was randomly oriented on the lab table in each trial to control for any unforeseen environmental effects.

\section{Experiment 1}

To determine the validity of the ideal free distribution as a tool for predicting patch use and the effect of predation risk, flies were allowed to choose between two patches with unequal amounts of sugar. Because flies did not have direct access to all sugar within a cube, surface area was used instead of mass to represent food availability. When altering proportions, sugar was cut into cubes with the appropriate surface area, while the total surface area was held approximately constant (mean of $665.5 \mathrm{~mm}^{2}$; range $\left.642.7-681.3 \mathrm{~mm}^{2}\right)$. This allowed for a total available surface area (5 sides of a cube) averaging $554.6 \mathrm{~mm}^{2}$ (range $535.5-567.8 \mathrm{~mm}^{2}$ ) in each trial. This total food level was determined to be appropriate as pilot experiments 
showed that at this level all flies were able to feed, but had to use both patches to do so. Also, the surface area of the sugar cubes did not decrease significantly over the $1 \mathrm{~h}$ trials so food was considered to be a nondiminishing resource. Three food distribution ratios were used based on available surface area; 25.5:74.5, 38.6:61.4, and 50:50.

In each trial, flies were placed into the tank and allowed to feed. The number of feeds (a feed being any time a fly touched the sugar) in each patch was recorded for $1 \mathrm{~h}$ Also, the number of flies feeding in each patch was recorded every five minutes for the entire hour, and summed. The proportion of flies feeding in each patch was then determined for both measurement methods and the correlation between these two values was determined. Food treatments were chosen randomly for each trial until six replicates were performed for each food distribution treatment.

\section{Experiment 2}

Spiders (Pardosa vancouveri Emerton) were used to create predation risk in patches. These predators were collected at two field sites in the BC lower mainland. They were considered appropriate predators for two reasons: (1) spiders were able to kill and consume live flies, and (2) flies tended to avoid spiders but would still eat in their presence if sufficient food was available.

To quantify the risk of predation, flies were presented with two patches of equal food but differing risks of predation. Each patch contained a $340.7 \mathrm{~mm}^{2}$ cube of sugar, with an available surface area (5 sides) of $283.9 \mathrm{~mm}^{2}$. Experiments were conducted at three different predation levels; 1,3 , and 5 spiders. The high-gloss surface of the tile partition was difficult for the spiders to scale, restricting them to the patch in which they were placed. Control experiments were also performed with no predators. The number of feeds in each patch was recorded for $1 \mathrm{~h}$ and used to determine the proportion of total feeds. The correlation between sampling methods found in Exper. 1 (see later) was used to convert the proportion of feeds to the proportion of flies feeding in each patch. Risk levels (and tank sides) were chosen randomly for each trial until 6 replicates for all risk levels (includng the control) had been performed.

\section{Experiment 3}

Results for Exper. 2 were used to calculate the energy equivalence of predation risk (EP) for all predation levels using the following formula 
(from Abrahams and Dill, 1989):

$$
\mathrm{EP}=\left(F_{r} / N_{r}\right)-\left(F_{s} / N_{s}\right)
$$

where $F_{\mathrm{r}}$ is the available surface area in the risky patch, $F_{\mathrm{s}}$ the available surface area in safe patch, $N_{\mathrm{r}}$ the number of flies in the risky patch, $N_{\mathrm{s}}$ the number of flies in the safe patch. These values were used to determine how much extra food must be added to the risky patch to offset the risk of predation and have flies distribute themselves equally between the two patches. In order to get $50 \%$ (or 15) flies to feed in the risky patch, the amount of available surface area needed in the risky patch was determined by the following formula (from Abrahams and Dill, 1989):

$$
F_{\mathrm{r}}^{\prime}=F_{\mathrm{s}}+(15 \times \mathrm{EP})
$$

Note that EP varies with predation risk level.

Although $F_{\mathrm{r}}^{\prime}$ was determined for all levels of predation, this prediction was only tested using the 3-predator treatment. In this experiment, $283.9 \mathrm{~mm}^{2}\left(F_{\mathrm{s}}\right)$ of sugar $(5$ sides$)$ was placed in the safe patch, while $F_{\mathrm{r}}^{\prime}$ was placed in the risky patch. The number of flies feeding in each patch was recorded every $5 \mathrm{~min}$ for $1 \mathrm{~h}$ and the total proportion of flies feeding in each patch was determined. Also, the number of feeds in each patch was recorded for $1 \mathrm{~h}$ to determine the correlation between these two measures in the presence of predators. Six replicates of this treatment were performed.

\section{RESULTS}

\section{Experiment 1}

The spatial distribution of flies (based on counts every $5 \mathrm{~min}$ ) was strongly influenced by the distribution of sugar between the two patches (Fig. 1), and the slope of the regression line describing the data (1.251, $R^{2}=0.356, P<0.01$ ) does not differ from the slope of 1.0 (input matching) predicted by the IFD. There is a significant difference among treatment groups (ANOVA, $P=0.023$ ), although Tukey HSD multiple comparison tests indicate that significant differences do not exist between adjacent groups (Fig. 1). Both the weak correlation between food and fly distribution and the lack of significant differences between adjacent treatment groups are undoubtedly due to the large variance in the data. Despite this, the general trend indicates that the IFD is an appropriate tool for determining the effects of predation risk on patch use in this system. 


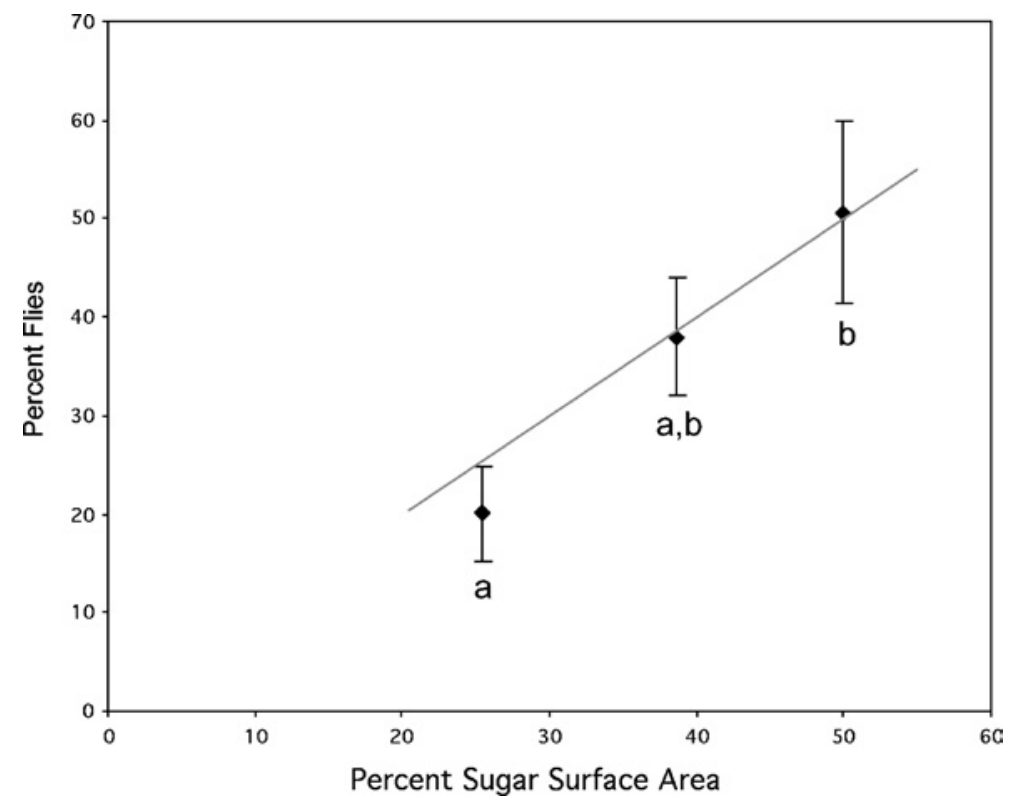

Fig. 1. The relationship between the distribution of food and the distribution of flies between two patches (based on counts every $5 \mathrm{~min}$ ). Error bars represent standard errors $(n=6)$. The predicted ideal free distribution (1:1 line) is shown for comparison. Points accompanied by the same letter are not significantly different (Tukey HSD test, $p>0.05$ ).

The two measurement methods (number of feeds in each patch for $1 \mathrm{~h}$ and total number of flies feeding in each patch every $5 \mathrm{~min}$ for $1 \mathrm{~h}$ ) used to determine patch use were highly correlated $\left(r^{2}=0.91, p<\right.$ $0.01)$. Percent flies can be calculated from $\%$ feeds from the equation: $\%$ flies $=-4.912+1.061$ ( $\%$ feeds $)$.

\section{Experiment 2}

Predation had a major effect on the distribution of flies among patches. When food was distributed equally among patches, fewer flies fed in the patch containing predators. As predation risk increased, use of the risky patch decreased (Fig. 2).

One-way ANOVA indicates a significant difference exists among groups $(P<0.001)$. However, results of Tukey HSD tests show no significant differences between the distributions of flies in adjacent treatment groups (Fig. 2). 


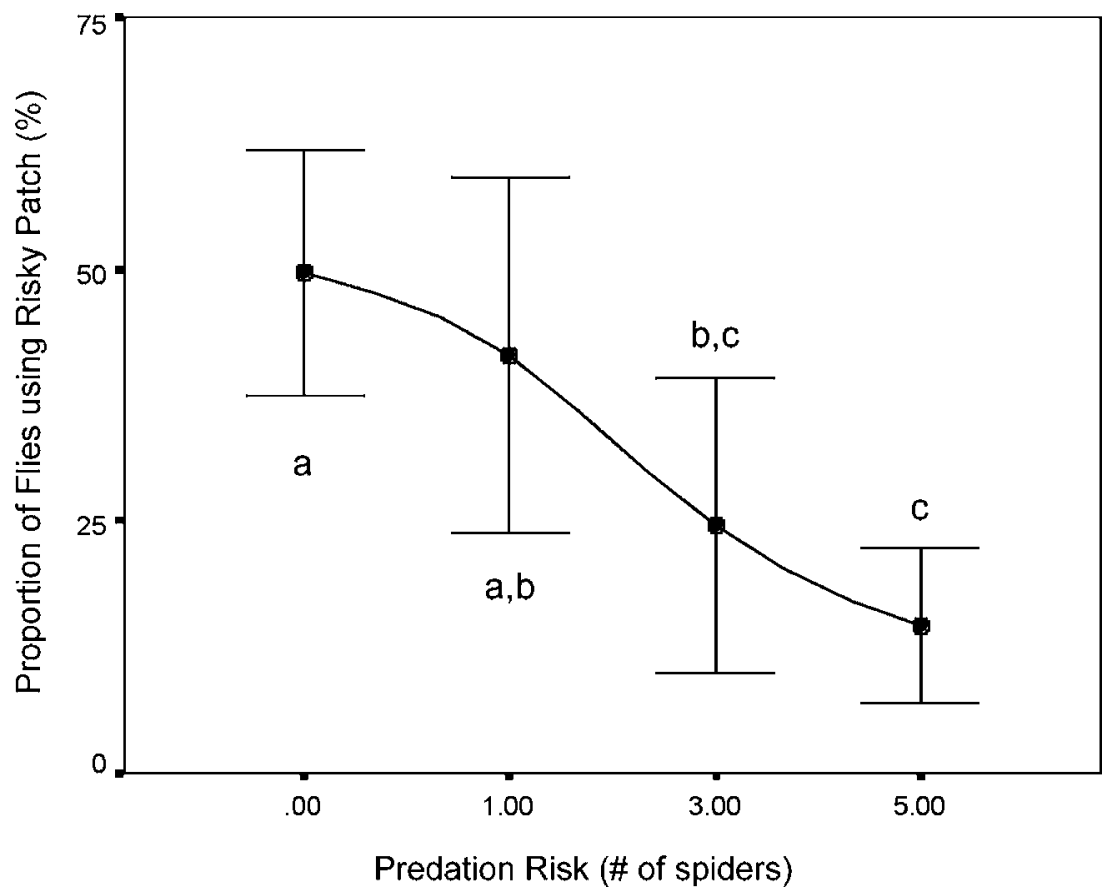

Fig. 2. The proportion of flies feeding in the risky patch as a function of predation risk (number of spiders present). Error bars represent standard errors $(n=6)$. Points accompanied by the same letter are not significantly different (Tukey HSD test, $p>0.05$ ).

\section{Experiment 3}

Table I presents the energy equivalence of predation risk (EP) and the amount of food necessary to offset the cost of predation $\left(F_{r}^{\prime}\right)$ as calculated from equations 1 and 2 for all predation treatments. When the calculated $F_{r}^{\prime}$ was added to the risky patch ( 3 spider treatment), flies distributed themselves approximately equally between both patches; the mean \% of flies in the risky patch was $47.61 \pm 3.81 \mathrm{SE}(n=6)$. Also, there was a significant difference between the proportion of flies feeding in the 3 -spider patch in Exper. 2, and the proportion feeding at this same risk level after the addition of $F_{r}^{\prime}(t$-test; $P=0.007)$.

\section{Measurement Methods}

The relationship between \% flies and \% feeds determined in Exper. 1, in the absence of a predator, was used to convert the data from Exper. 2, 
Table I. Energy Equivalence of Predation Risk (EP) and Amount of Food Necessary to Offset Risk of Predation $\left(F_{r}^{\prime}\right)$ for Three Levels of Predation Risk

\begin{tabular}{cccr}
\hline Predation risk (\# spiders) & EP $\left(\mathrm{mm}^{2}\right)$ & EP per spider $\left(\mathrm{mm}^{2}\right)$ & $F_{r}^{\prime}\left(\mathrm{mm}^{2}\right)$ \\
\hline 1 & 6.70 & 6.70 & 384.37 \\
3 & 25.94 & 8.65 & 673.04 \\
5 & 53.59 & 10.72 & 1087.70 \\
\hline
\end{tabular}

when a predator was present. It was therefore important to determine the relationship for the latter scenario. This was done in Exper. 3, yielding the predictive equation: \% flies $=8.746+0.817$ (\% feeds) $\left(r^{2}=0.90 ; p<0.01\right)$. Neither the slope nor the intercept is significantly different from those of the predator-free relationship (see Exper. 1 Results), justifying that equation's use to transform the Exper. 2 data. The data from both experiments were combined in a single regression model, conditioned through the origin. The line of best fit (Fig. 3) is described by the equation $\left(r^{2}=0.90, n=24\right.$, $p<0.001)$ :

$$
\% \text { flies }=0.9674(\% \text { feeds })
$$

Using this equation throughout would not alter our qualitative conclusions, and would affect the quantitative estimates of EP only slightly.

\section{DISCUSSION}

In general, our results demonstrate that foraging female houseflies tend to distribute themselves as predicted by the ideal free distribution. However, results from all experiments show a large amount of variation, possibly resulting from violations of IFD assumptions. One violation may result from superior competitors preventing "free" access to defendable resources, often suggested as a reason for deviations from the IFD (Grand and Grant, 1994). Animals may also be constrained in their ability to correctly evaluate their environment, violating the "ideal" assumption. Abrahams (1989) gave this as a reason for initial deviation from IFD in female guppies (Poecilia reticulata), which conformed more closely to the IFD with increased exposure to similar environments.

In several trials during our experiment, it took some time for flies to locate sugar in one or both patches, with the sugar located first being exploited by more flies than expected until the other was discovered. This may have led to deviations from IFD in early phases of some trails and consequently affected the results. Collins and Bell (1996) found that the presence of a fly-like visual stimulus greatly increased the ability of $M$. domestica to locate food (sucrose drops), a possible adaptation to both mate location and 


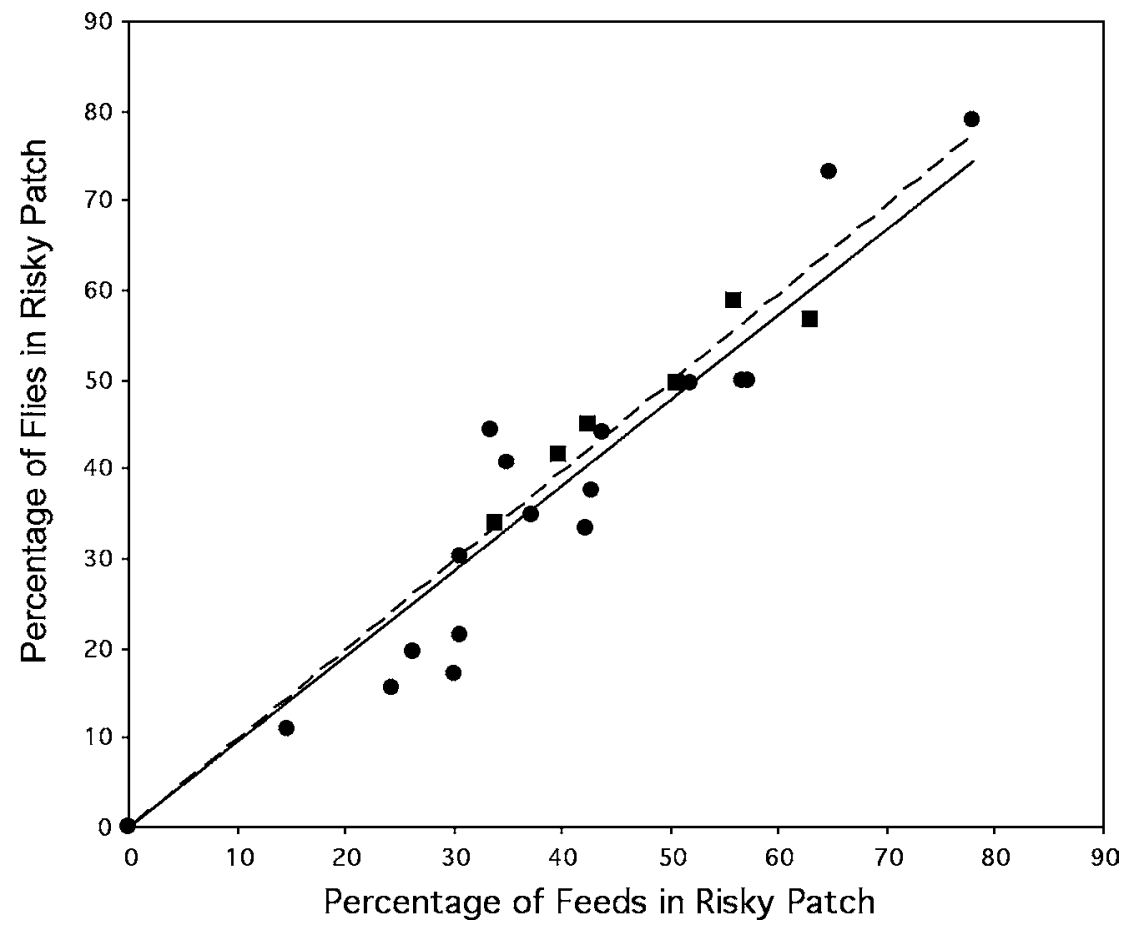

Fig. 3. Correlation between two patch use measurement methods in predator-present (Exper. 3; squares) and predator-absent (Exper. 1; circles) treatments. Proportion of feeds was determined by proportion of times flies touched the sugar cube in $1 \mathrm{~h}$, while proportion of flies was determined by the proportion of flies feeding in each patch, measured every $5 \mathrm{~min}$. for $1 \mathrm{~h}$ and summed. The slopes of the two regressions (both conditioned through the origin) are not significantly different from one another; the regression equation for the pooled data is reported in the text.

resource finding. Thus, placement of a fly-like object next to sugar in both patches may increase the likelihood of resource finding, leading to better knowledge of the environment and increased conformity to IFD.

Our results demonstrate that predation risk can be measured in terms of energy, providing a surrogate measure for the effect of predation risk on fitness in the absence of a direct fitness measure. When exposed to two patches, one with an associated risk of predation, flies showed increased preference for predator-free patches as predation risk increased. In all risk treatments some flies continued to feed in the risky patch despite the presence of predators. Because IFD was found to be a valid assumption in this system, results suggest that flies were trading-off predation risk for increased access to food. Presumably all flies obtained equal fitness in terms of the resource-risk trade-off. The addition of food to the risky patch was 
successful in offsetting the risk of predation by providing flies feeding in the risky patch with greater access to food, and increasing the net benefit of the risky patch to equal that of the safe patch. Similar results were found in experiments using guppies (Abrahams and Dill, 1989) and gerbils (Kotler and Blaustein, 1995; Abramsky et al., 2002).

In several experiments, patch use was determined both by counting the number of feeds in each patch over a $1 \mathrm{~h}$ period and by counting the number of flies feeding in each patch every $5 \mathrm{~min}$ for $1 \mathrm{~h}$. In both predatorabsent and predator-present conditions, these two measures appeared to be strongly correlated. Feeds are far easier to measure, especially when there is a large fly population, and this metric is recommended to anyone wishing to do similar studies.

It is interesting to note how the energy equivalence of predation risk (EP) changes with increased risk. Not only did the EP increase with increasing risk, but EP per spider also increased with number of spiders (see Table I). One possible explanation for this finding is that vigilance behavior takes up an increasing proportion of each fly's time as the number of predators increases. Also, escape from one predator may lead to increased likelihood of interaction with another as predator density increases. As only three predator treatments were used in this experiment, these hypotheses are speculative and should be explored in future experiments with a greater number of predator treatments.

\section{ACKNOWLEDGMENTS}

We thank Dr. Kris Rothley (assistance with spider collection), Max Salomon (spider identification), Kevin Lam (providing houseflies), and Ian Gordon and Mark Wong (assistance with experimental logistics and statistical analysis). LMD's research is supported by NSERC Canada Discovery Grant A6869.

\section{REFERENCES}

Abrahams, M. V. (1989). Foraging guppies and the ideal free distribution: The influence of information on patch choice. Ethology 82: 116-126.

Abrahams, M. V., and Dill, L. M. (1989). A determination of the energetic equivalence of the risk of predation. Ecology 70: 999-1007.

Abramsky, Z., Rosenzweig, M. L., and Subach, A. (2002). The costs of apprehensive foraging. Ecology 83: 1330-1340.

Collins, R. D., and Bell, W. J. (1996). Enhancement of resource finding efficiency by visual stimulation in Musca domestica (Diptera: Muscidae). J. Kans. Ent. Soc. 69: 204-207.

Courtney, S. P., and Parker, G. A. (1985). Mating behaviour of the tiger blue butterfly (Tarcus theophrastus): competitive mate-searching when not all females are captured. Behav. Ecol. Sociobiol. 17: 213-221. 
Diaz, M., Illera, J. C., and Atienza, J. C. (1998). Food resource matching by foraging tits Parus spp. during spring-summer in a Mediterranean mixed forest; evidence for an ideal free distribution. Ibis 140: 654-660.

Fretwell, S. D., and Lucas, H. L. (1970). On territorial behavior and factors influencing habitat distribution in birds. I.Theoretical development. Acta Biotheor. 19: 16-36.

Gotceitas, V., and Colgan, P. (1991). Assessment of patch profitability and ideal free distribution: The significance of sampling. Behavior 199: 65-76.

Grand, T. C., and Grant, J. W. A. (1994). Spatial predictability of resources and the ideal free distribution in convict cichlids, Cichlasoma nigrofasciatum. Anim. Behav. 48: 909-919.

Kotler, B. P., and Blaustein, L. (1995). Titrating food and safety in a heterogeneous environment: when are the risky and safe patches of equal value? Oikos 74: 251-258.

Kraft, J. R., and Baum, W. M. (2001). Group choice: The ideal free distribution of human social behavior. J.Exp. Anal. Behav. 76: 21-42.

Lamb, A. E., and Ollason, J. G. (1993). Foraging wood-ants Formica aquilonia Yarrow (Hymenoptera: Formicidae) tend to adopt the ideal free distribution. Behav. Proc. 28: 189-198.

Larsson, P. (1997). Ideal free distribution in Daphnia? Are daphnids able to consider both the food patch quality and the position of competitors? Hydrobiologia 360: 143-152.

Lima, S. L., and Dill, L. M. (1990). Behavioral decisions made under the risk of predation: A review and prospectus. Can. J. Zool. 68: 619-640.

MacArthur, R. H., and Pianka, E. R. (1966). On optimal use of a patchy environment. Amer. Nat. 100: 603-609.

Madden, G. J., Peden, B. F., and Yamaguchi, T. (2002). Human group choice: Discrete-trial and free-operant tests of the ideal free distribution. J. Exper. Anal. Behav. 78: 1-15.

Parker, G. A. (1978). Searching for mates. In Krebs, J. R., and Davies, N. B. (eds.), Behavioural Ecology: An Evolutionary Approach. Blackwell, Oxford, pp. 214-244.

Stephens, D. W., and Krebs, J. R. (1986). Foraging Theory. Princeton University Press, Princeton, New Jersey. 